\title{
Mesenchymal stem cell therapy for acute and chronic pancreatitis
}

\author{
Kazumichi Kawakubo $^{1}$ (D) Shunsuke Ohnishi ${ }^{1} \cdot$ Masaki Kuwatani $^{2}$ • \\ Naoya Sakamoto ${ }^{1}$
}

Received: 30 April 2017 / Accepted: 12 June 2017/Published online: 23 June 2017

(C) Japanese Society of Gastroenterology 2017

\begin{abstract}
Mesenchymal stem cells (MSCs) have attracted attention as a cell source for regenerative medicine. In particular, MSCs have an anti-inflammatory effect by secreting several kinds of bioactive molecules. MSC therapy is now being applied to various gastrointestinal diseases, such as graft-versus-host disease, inflammatory bowel disease, and liver cirrhosis. Therefore, MSC therapy has the potential to be a novel treatment for acute and chronic pancreatitis by suppressing inflammation. Several studies have investigated the effect of MSC therapy on acute and chronic pancreatitis, but the underlying mechanisms remain unknown. In this review, we summarize the present status of MSC therapy for acute and chronic pancreatitis.
\end{abstract}

Keywords Mesenchymal stem cells - Acute pancreatitis . Chronic pancreatitis $\cdot$ Regenerative medicine $\cdot$ Cell therapy

\section{Introduction}

Mesenchymal stem cells (MSCs) are multipotent cells that occur in several tissues, such as bone marrow, adipose tissue, and fetal membranes [1]. MSCs have been investigated for regenerative medicine because of their immunomodulatory effects on the secretion of a variety of

Kazumichi Kawakubo

kkawakubo-gi@umin.ac.jp

1 Department of Gastroenterology and Hepatology, Faculty of Medicine and Graduate School of Medicine, Hokkaido University, Kita15 Nishi7, Kita-ku, Sapporo 060-8638, Japan

2 Division of Endoscopy, Hokkaido University Hospital, Kita14 Nishi5, Kita-ku, Sapporo 060-8648, Japan anti-inflammatory molecules [2]. Because MSCs do not have the major histocompatibility complex class II antigen, rejection does not occur, even after allogenic transplantation. The effect of MSC transplantation has been investigated in several gastrointestinal diseases in animal models [3-5] and in human trials [6].

Acute pancreatitis (AP) and chronic pancreatitis (CP) are inflammatory disorders of the pancreas [7, 8]. The antiinflammatory effect of MSCs is considered a promising treatment strategy for AP and CP [9-11]. In this review article, we summarize the current evidence of MSC therapy for $\mathrm{AP}$ and $\mathrm{CP}$ and their underlying mechanisms.

\section{Mesenchymal stem cells}

MSCs were first isolated and characterized in 1976 [12]. They possess a self-renewal ability and can differentiate into cells of multiple lineages, including mesoderm lineages, such as chondrocytes, osteocytes and adipocytes, and ectodermic and endodermic cells [1]. MSCs can be isolated from the bone marrow, adipose tissue, and umbilical cord, and are easily expanded in vitro. In general, MSCs must (a) remain plastic-adherent under standard culture conditions; (b) express CD105, CD73, and CD90, but not CD45, CD34, CD14, CD11b, CD79a CD19, or HLA-DR; and (c) differentiate into osteoblasts, adipocytes, and chondrocytes in vitro [13]. Cell surface markers were confirmed by flow cytometric analysis. Multilineage differentiation was induced by incubation in adipogenic, osteogenic, and chondrogenic media in vitro.

In 2004, Le Blanc et al. reported that a graft-versus-host disease patient was successfully treated by transplantation of MSCs [14]. Since then, several clinical trials of MSCs have been reported. At first, MSC therapy focused on their 
capacity for multilineage differentiation. However, several studies revealed that a large fraction of systemically infused MSCs were trapped within the lungs as emboli owing to their large size [15]. Furthermore, MSC therapy was shown to suppress inflammation, apoptosis, and fibrosis in numerous disease models without MSC differentiation and engraftment in the injured tissue [5, 16]. Therefore, clinical trials have shifted toward utilizing the biological functions of MSCs through trophic mechanisms and from local to systemic administration [17-21]. This is the main advantage of MSC therapy over embryonic stem (ES) cells and inducible pluripotent stem (iPS) cells, which require differentiation into specific cell lineages and engraftment in the target tissue; moreover, ES and iPS cells can form teratomas [22, 23].

\section{MSC therapy for acute pancreatitis}

Table 1 summarizes MSC therapy for AP. The sources of MSCs vary and include bone marrow, umbilical cord, adipose tissue, and fetal membranes. Allogenic and autologous MSC transplantation have been performed. MSC therapy for AP was first described in 2009 by Jung et al. [24]. They evaluated the effect of human bone marrowderived MSC transplantation in rats with mild and severe AP. MSCs attenuate histological scoring in the pancreas and reduce inflammatory cytokine expression in the pancreas as well as serum levels of transforming growth factor (TGF)- $\beta$, tumor necrosis factor (TNF)- $\alpha$, and interferon (IFN) $-\gamma$. They also found that the number of Foxp3+ positive regulatory $\mathrm{T}$ cells increases in the lymph node and pancreas. Another study reported that MSCs decrease the number of CD3-positive $\mathrm{T}$ cells and increase the number of Fox-P3 positive $\mathrm{T}$ cells in the pancreas [25]. Several studies have reported that MSCs suppress serum and pancreatic levels of pro-inflammatory cytokines and increase anti-inflammatory cytokines [24, 26]. Yang et al. reported that the anti-inflammatory effect of MSC transplantation is time- and dose-dependent [27]. MSCs transplanted immediately after induction of AP have a better anti-inflammatory effect than those transplanted several hours after initiation. MSCs themselves have anti-inflammatory properties and serve as a vector for gene therapy. Hua et al. reported that angiopoietin-1 transfected MSCs reduce inflammation in rats with AP more than MSCs do alone [28] (Fig. 1).

The small intestine is damaged in AP because microcirculation is altered following fluid loss, hypovolemia, splanchnic vasoconstriction, and ischemia-reperfusion injury. Tu et al. reported that MSCs improve intestinal injury during severe AP by increasing aquaporin (AQP)-1 expression, reducing mucosal permeability of the intestine, promoting the recovery of intestinal epithelial cells, and maintaining integrity of the intestinal mucosal barrier [29]. Infusing MSCs reduces serum levels of malondialdehyde, superoxide dismutase levels, interleukin (IL)-6, IL-10, and TNF- $\alpha$. Transplanting MSCs attenuates intestinal mucosal injury and promotes mucosal repair in rats with severe AP [30]. Another study also showed that MSCs reduce damage to the small intestinal capillary endothelial barrier by increasing AQP-1 expression in the small intestine [31].
Table 1 MSC for animal models of acute pancreatitis

\begin{tabular}{llllll}
\hline Authors & MSCs source & Routes & Dose (cells) & Animal & Induction \\
\hline Jung [24] & Human BM & IV & $1 \times 10^{6}$ & Rat & Cerulein, 3\% TCA \\
Tu [30] & Rat BM & IV & $2 \times 10^{7} / \mathrm{kg}$ & Rat & Deoxy STC \\
Meng [26] & Human UC & IV & $1 \times 10^{7} / \mathrm{kg}$ & Rat & $3 \%$ TCA \\
Yang [27] & Human UC & IV & $5 \times 10^{6} / \mathrm{kg}$ & Rat & 3\% TCA \\
Hua [28] & Human UC & IV & $2 \times 10^{6}$ & Rat & 3\% TCA \\
Jung [32] & Human BM & IV & $1 \times 10^{6}$ & Rat & Cerulein + LPS \\
Qian [34] & BM & IV & $1 \times 10^{7} / \mathrm{kg}$ & Rat & 3\% TCA \\
Yin [36] & Rat BM & IV & $1 \times 10^{6}$ & Rat & L-Arg \\
He [35] & Human BM & IV & $2 \times 10^{6}$ & Mouse & 2\% TCA \\
Kawakubo [33] & Rat FM & IV & $1 \times 10^{6}$ & Rat & $4 \%$ TCA \\
Kim [25] & Canine AT & IV & $1 \times 10^{7} / \mathrm{kg}$ & Rat & $3 \%$ TCA \\
Yin [36] & Rat BM & IV & NA & Mouse & Cerulein \\
& & & & Rat & $4 \%$ TCA \\
Zhao [37] & Rat BM & IV & $5-7 \times 10^{7}$ & Rat & $3 \%$ TCA \\
Lu [31] & Rat BM & IV & $1 \times 10^{6}$ & Rat & $5 \%$ TCA \\
\hline
\end{tabular}

$M S C$ mesenchymal stem cell, $B M$ bone marrow, $T C A$ taurocholate, $U C$ umbilical cord, $F M$ fetal membrane, $A T$ adipose tissue, $N A$ not available, $I V$ intravenous 
Fig. 1 Presumed mechanisms of mesenchymal stem cell (MSC) therapy for acute and chronic pancreatitis. MSCs secrete several kinds of bioactive molecules that affect several targets. MSCs migrate and home to the pancreas

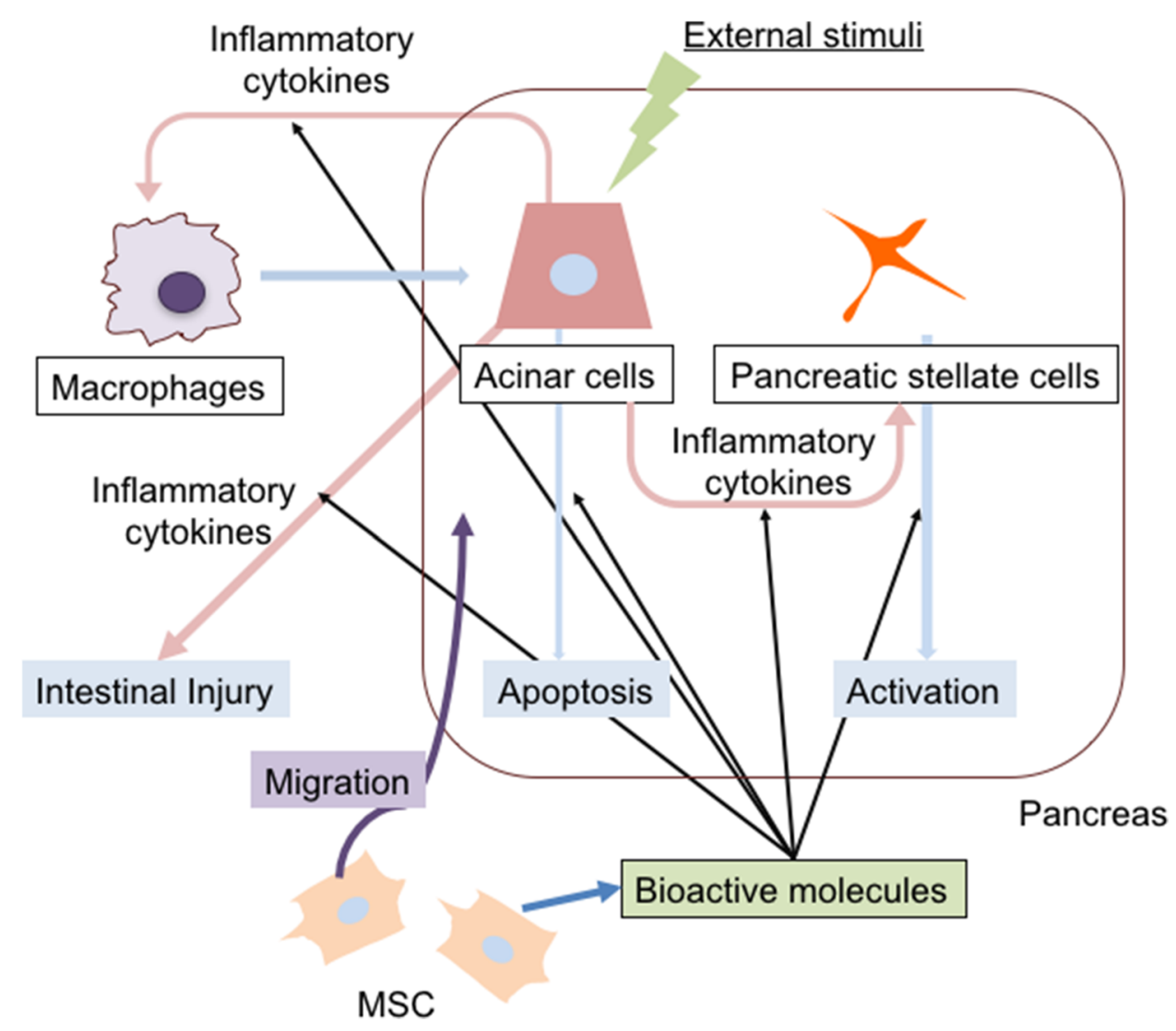

Another mechanism of MSCs for suppressing inflammation in AP is the anti-apoptosis effect [32, 33]. MSCs promote repair and angiogenesis via the stromal cell derived factor (SDF)-1 $\alpha / \mathrm{C}-\mathrm{X}-\mathrm{C}$ chemokine receptor type 4 (CXCR4) axis [34]. The precise mechanism remains controversial, but several bioactive molecules secreted by MSCs have important roles. He et al. reported that tumor necrosis factor- $\alpha$ stimulated gene/protein 6 (TSG6) is secreted by MSCs [35]. Yin et al. showed that microvesicles from MSCs attenuate injury due to AP [36]. However, several studies have described that MSCs can migrate and implant in several organs including the pancreas $[24,35,37]$. Further studies are necessary to investigate the underlying mechanisms of MSCs in inflammation due to AP.

\section{MSC therapy for chronic pancreatitis}

In contrast to AP, only a few studies have investigated the effects of MSCs in a CP model (Table 2). Zhou et al. reported that umbilical cord-derived MSCs reduce pancreatic fibrosis in a dibutyltin dichloride-induced rat model of $\mathrm{CP}$ by suppressing inflammatory cytokines in the pancreas [38]. We have demonstrated that human amnion- derived MSCs decrease monocyte chemotactic protein-1 (MCP-1) expression in the pancreas [33]. Pancreatic stellate cells are responsible for fibrosis in $\mathrm{CP}$. We showed that conditioned medium obtained from human amnion-derived MSCs suppresses MCP-1 and IL-8 expression in pancreatic stellate cells. The precise mechanisms and roles of MSCs in $\mathrm{CP}$ remain unknown, and further studies are warranted in the future.

\section{Perspectives}

Numerous animal model studies have documented the therapeutic potential of MSCs and their safety and efficacy in vivo. However, their clinical application was hampered by several remaining issues, i.e., the quality of MSCs and their non-uniform effect due to the requirement for living donors and culture. Standardized methods to control the quality of MSC therapy should be established. The factors secreted by MSCs responsible for their effects are unknown and their kinetics should be elucidated. The optimal conditions for homing and cytokine production by MSCs should be elucidated. The optimal timing and dosing of MSC administration should be determined and the most responsive patient groups should be identified. 
Table 2 MSC for animal model of chronic pancreatitis

\begin{tabular}{llllll}
\hline Authors & MSCs source & Routes & Dose (cells) & Animal & Induction \\
\hline Zhou [38] & Rat UC & IV & $2 \times 10^{6} / \mathrm{kg}$ & Rat & DBTC \\
Kawakubo [33] & Human amnion & IV & $1 \times 10^{6}$ & Rat & DBTC \\
\hline
\end{tabular}

$M S C$ mesenchymal stem cell, $B M$ bone marrow, DBTC dibutyltin dichloride, $U C$ umbilical cord, $I V$ intravenous

\section{Conclusions}

No human clinical trials have used MSCs to treat AP or CP. However, animal trials have revealed that MSC transplantation would be a novel therapy for AP and CP.

\section{Compliance with ethical standards}

Conflict of interest The authors declare that they have no conflict of interest.

\section{References}

1. Pittenger MF, Mackay AM, Beck SC, et al. Multilineage potential of adult human mesenchymal stem cells. Science. 1999;284:143-7.

2. Uccelli A, Moretta L, Pistoia V. Mesenchymal stem cells in health and disease. Nat Rev Immunol. 2008;8:726-36.

3. Kubo K, Ohnishi S, Hosono H, et al. Human amnion-derived mesenchymal stem cell transplantation ameliorates liver fibrosis in rats. Transplant Direct. 2015;1:e16.

4. Onishi R, Ohnishi S, Higashi R, et al. Human amnion-derived mesenchymal stem cell transplantation ameliorates dextran sulfate sodium-induced severe colitis in rats. Cell Transplant. 2015;24:2601-14.

5. Ono M, Ohnishi S, Honda M, et al. Effects of human amnionderived mesenchymal stromal cell transplantation in rats with radiation proctitis. Cytotherapy. 2015;17:1545-59.

6. Trounson A, McDonald C. Stem cell therapies in clinical trials: progress and challenges. Cell Stem Cell. 2015;17:11-22.

7. Braganza JM, Lee SH, McCloy RF, et al. Chronic pancreatitis. Lancet. 2011;377:1184-97.

8. Frossard J-L, Steer ML, Pastor CM. Acute pancreatitis. Lancet. 2008;371:143-52.

9. Ito $\mathrm{T}$, Ishiguro $\mathrm{H}$, Ohara $\mathrm{H}$, et al. Evidence-based clinical practice guidelines for chronic pancreatitis 2015. J Gastroenterol. 2016;51:85-92.

10. Masamune A, Kikuta K, Nabeshima T, et al. Nationwide epidemiological survey of early chronic pancreatitis in Japan. J Gastroenterol. 2017. doi:10.1007/s00535-017-1311-8.

11. Tsuji Y, Takahashi N, Isoda H, et al. Erratum to: early diagnosis of pancreatic necrosis based on perfusion CT to predict the severity of acute pancreatitis. J Gastroenterol. 2017. doi:10.1007/ s00535-017-1343-0.

12. Friedenstein AJ, Gorskaja JF, Kulagina NN. Fibroblast precursors in normal and irradiated mouse hematopoietic organs. Exp Hematol. 1976;4:267-74.

13. Dominici M, Le Blanc K, Mueller I, et al. Minimal criteria for defining multipotent mesenchymal stromal cells. The International Society for Cellular Therapy position statement. Cytotherapy. 2006;8:315-7.

14. Le Blanc K, Rasmusson I, Sundberg B, et al. Treatment of severe acute graft-versus-host disease with third party haploidentical mesenchymal stem cells. Lancet. 2004;363:1439-41.
15. Barbash IM, Chouraqui P, Baron J, et al. Systemic delivery of bone marrow-derived mesenchymal stem cells to the infarcted myocardium: feasibility, cell migration, and body distribution. Circulation. 2003;108:863-8.

16. Lee RH, Pulin AA, Seo MJ, et al. Intravenous hMSCs improve myocardial infarction in mice because cells embolized in lung are activated to secrete the anti-inflammatory protein TSG-6. Cell Stem Cell. 2009;5:54-63.

17. Miyamoto S, Ohnishi S, Onishi R, et al. Therapeutic effects of human amnion-derived mesenchymal stem cell transplantation and conditioned medium enema in rats with trinitrobenzene sulfonic acid-induced colitis. Am J Transl Res. 2017;9:940-52.

18. Mizushima T, Ohnishi S, Hosono $\mathrm{H}$, et al. Oral administration of conditioned medium obtained from mesenchymal stem cell culture prevents subsequent stricture formation after esophageal submucosal dissection in pigs. Gastrointest Endosc. 2017. doi:10. 1016/j.gie.2017.01.024.

19. Nagaishi K, Arimura Y, Fujimiya M. Stem cell therapy for inflammatory bowel disease. J Gastroenterol. 2015;50:280-6.

20. Terai S, Tsuchiya A. Status of and candidates for cell therapy in liver cirrhosis: overcoming the "point of no return" in advanced liver cirrhosis. J Gastroenterol. 2017;52:129-40.

21. Watanabe S, Arimura Y, Nagaishi K, et al. Conditioned mesenchymal stem cells produce pleiotropic gut trophic factors. J Gastroenterol. 2014;49:270-82.

22. Takahashi K, Yamanaka S. Induction of pluripotent stem cells from mouse embryonic and adult fibroblast cultures by defined factors. Cell. 2006;126:663-76.

23. Thomson JA, Itskovitz-Eldor J, Shapiro SS, et al. Embryonic stem cell lines derived from human blastocysts. Science. 1998;282:1145-7.

24. Jung KH, Song SU, Yi T, et al. Human bone marrow-derived clonal mesenchymal stem cells inhibit inflammation and reduce acute pancreatitis in rats. Gastroenterology. 2011;140:9981008.

25. Kim HW, Song WJ, Li Q, et al. Canine adipose tissue-derived mesenchymal stem cells ameliorate severe acute pancreatitis by regulating $\mathrm{T}$ cells in rats. J Vet Sci. 2016;17:539-48.

26. Meng HB, Gong J, Zhou B, et al. Therapeutic effect of human umbilical cord-derived mesenchymal stem cells in rat severe acute pancreatitis. Int J Clin Exp Pathol. 2013;6:2703-12.

27. Yang B, Bai B, Liu CX, et al. Effect of umbilical cord mesenchymal stem cells on treatment of severe acute pancreatitis in rats. Cytotherapy. 2013;15:154-62.

28. Hua J, He ZG, Qian DH, et al. Angiopoietin-1 gene-modified human mesenchymal stem cells promote angiogenesis and reduce acute pancreatitis in rats. Int J Clin Exp Pathol. 2014;7:3580-95.

29. Tu XH, Huang SX, Li WS, et al. Mesenchymal stem cells improve intestinal integrity during severe acute pancreatitis. Mol Med Rep. 2014;10:1813-20.

30. Tu XH, Song JX, Xue XJ, et al. Role of bone marrow-derived mesenchymal stem cells in a rat model of severe acute pancreatitis. World J Gastroenterol. 2012;18:2270-9.

31. Lu F, Wang F, Chen Z, et al. Effect of mesenchymal stem cells on small intestinal injury in a rat model of acute necrotizing pancreatitis. Stem Cell Res Ther. 2017;8:12. 
32. Jung KH, Yi T, Son MK, et al. Therapeutic effect of human clonal bone marrow-derived mesenchymal stem cells in severe acute pancreatitis. Arch Pharm Res. 2015;38:742-51.

33. Kawakubo K, Ohnishi S, Fujita H, et al. Effect of fetal membrane-derived mesenchymal stem cell transplantation in rats with acute and chronic pancreatitis. Pancreas. 2016;45:707-13.

34. Qian D, Gong J, He Z, et al. Bone marrow-derived mesenchymal stem cells repair necrotic pancreatic tissue and promote angiogenesis by secreting cellular growth factors involved in the SDF$1 \mathrm{alpha} / \mathrm{CXCR} 4$ axis in rats. Stem Cells Int. 2015;2015:306836.

35. He Z, Hua J, Qian D, et al. Intravenous hMSCs ameliorate acute pancreatitis in mice via secretion of tumor necrosis factor-alpha stimulated gene/protein 6. Sci Rep. 2016;6:38438.
36. Yin $\mathrm{G}, \mathrm{Hu} \mathrm{G}$, Wan $\mathrm{R}$, et al. Role of microvesicles from bone marrow mesenchymal stem cells in acute pancreatitis. Pancreas. 2016;45:1282-93.

37. Zhao H, He Z, Huang D, et al. Infusion of bone marrow mesenchymal stem cells attenuates experimental severe acute pancreatitis in rats. Stem Cells Int. 2016;2016:7174319.

38. Zhou CH, Li ML, Qin AL, et al. Reduction of fibrosis in dibutyltin dichloride-induced chronic pancreatitis using rat umbilical mesenchymal stem cells from Wharton's jelly. Pancreas. 2013;42:1291-302. 